\title{
L'art des «pierres à cerfs» de Mongolie
} Jérôme Magail

\section{Citer ce document / Cite this document :}

Magail Jérôme. L'art des «pierres à cerfs» de Mongolie. In: Arts asiatiques, tome 60, 2005. pp. 172-180;

doi : https://doi.org/10.3406/arasi.2005.1539

https://www.persee.fr/doc/arasi_0004-3958_2005_num_60_1_1539

Fichier pdf généré le 21/04/2018 


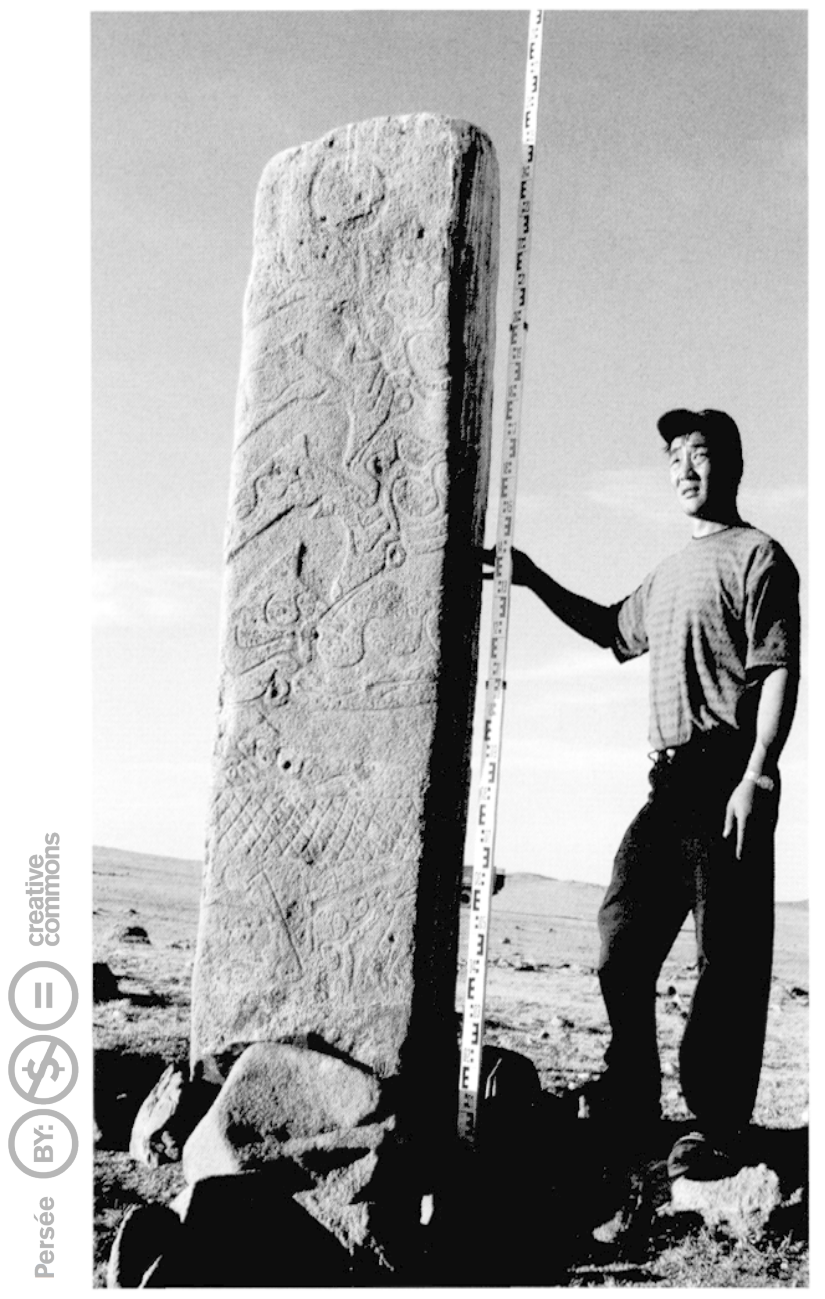

Fig. 1a-b

Stèle 4 de Gol Mod. Granit, Dim. 2,10 x 0,6 x 0,3 m, Urtyn Am, Vallée de Khujirbulan. (Phot. et schéma J. Magail.)
L'aire géographique où ont été érigées ces stèles correspond à une zone qui couvre un tiers de l'empire Xiongnu ${ }^{3}$. Le professeur D. Tseevendorj a ainsi évalué à 700 le nombre de «pierres à cerfs" réparties dans dix provinces mongoles, en Bouriatie et dans la région russe de Touva ${ }^{4}$.

La hauteur moyenne de ces centaines de monolithes est de 1,8 m. Certains peuvent dépasser les $4 \mathrm{~m}$, comme celui du site archéologique de Uushig dans la province du Hövsgöl, qui atteint 4,8 m de haut. La plupart de ces stèles ont une section rectangulaire et présentent deux faces larges de $60 \mathrm{~cm}$ en moyenne, et deux faces étroites d'environ $30 \mathrm{~cm}$.

Sur les stèles les plus complètes on relève des cervidés, des armes, des astres et des motifs géométriques (fig. 3a-b). La répartition des thèmes iconographiques et leur typologie semblent obéir aux mêmes conventions sur tous les monuments. Les stèles de Gol Mod, qui ne dérogent pas à la règle, appartiennent à la typologie la plus ancienne selon la classification établie par E. Novgorodova. En leur sommet, deux cercles de tailles différentes semblent représenter le soleil et la lune $e^{5}$. Le soleil est parfois rayonnant comme figuré sur la stèle conservée au musée de la ville de Tsetserleg (fig. 4). Au-dessous des astres, dans la partie médiane, des cerfs aux longs bois enroulés, s'élancent vers le haut ou vers le bas de la stèle. Quant à la base du monument, elle est marquée de boucliers,
$\mathrm{Au}$ cours de la transition entre les périodes de l'âge du bronze et de l'âge du fer, les tribus de Haute Asie ont laissé de superbes stèles gravées, connues sous le terme de "pierres à cerfs» (fig. 1a-b). Elles ont été répertoriées de la Mongolie orientale à l'Altaï, du nord du Gobi à la Transbaïkalie. Cinq de ces monuments appartiennent à un cimetière proche de la nécropole Xiongnu de Gol Mod', qui est située à $450 \mathrm{~km}$ à l'ouest de la capitale Oulan-Bator (fig. 2) et étudiée par la Mission archéologique française en Mongolie ${ }^{2}$. Les «pierres à cerfs» ont été édifiées deux ou trois siècles avant l'avènement de la confédération tribale qui forma le vaste empire Xiongnu au III" s. av. J.-C. Les mours, coutumes et cosmologie des Xiongnu sont-elles en partie héritées des nomades, pasteurs et guerriers du I‘r millénaire?

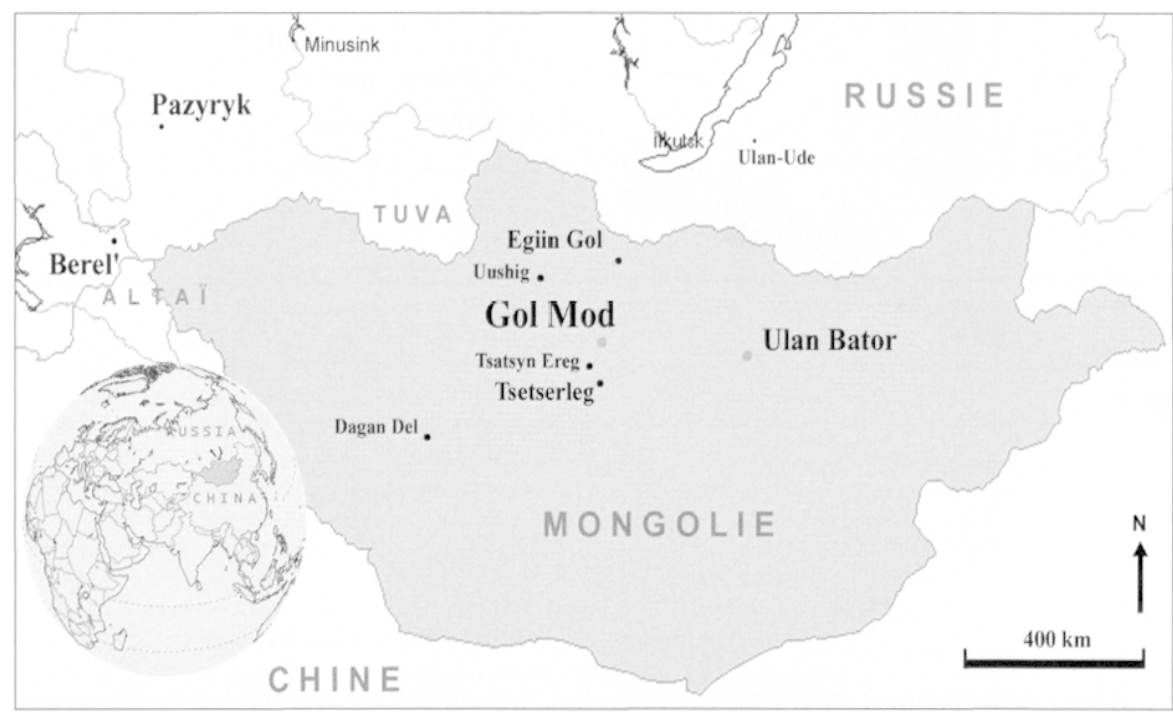

Fig. 2

Carte de la Mongolie et des régions limitrophes. 

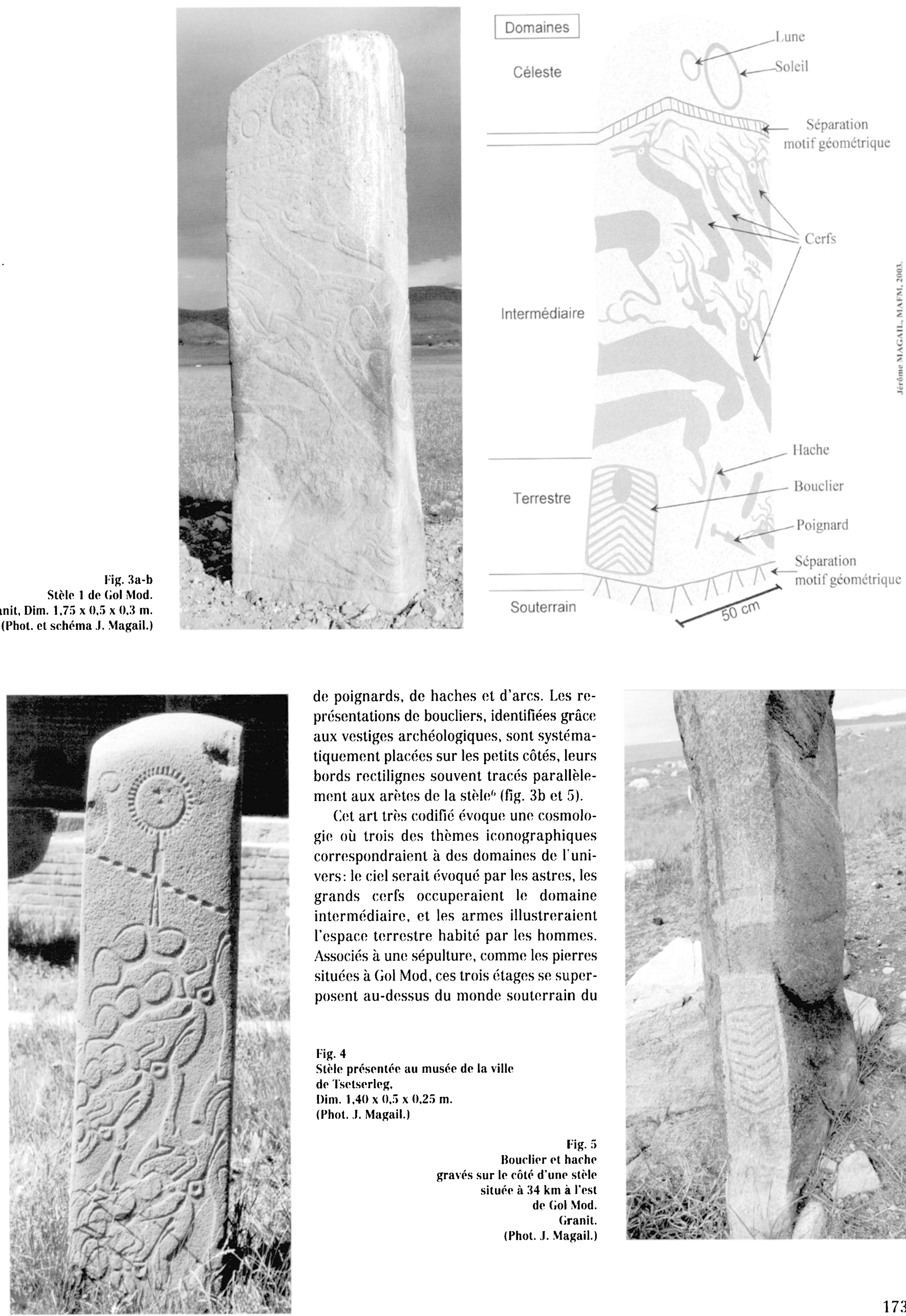

de poignards, de haches et d'arcs. Les représentations de boucliers, identifiées grâce aux vestiges archéologiques, sont systématiquement placées sur les petits côtés, leurs bords rectilignes souvent tracés parallèlement aux arètes de la stèle" (fig. 3b et 5).

Cet art très codifié évoque une cosmologie où trois des thèmes iconographiques correspondraient à des domaines de l'univers: le ciel serait évoqué par les astres, les grands cerfs occuperaient le domaine intermédiaire, et les armes illustreraient l'espace terrestre habité par les hommes. Associés à une sépulture, comme les pierres situées à (jol Mod, ces trois étages se superposent au-dessus du monde souterrain du

Fig. 4

Stèle présentée au musée de la ville de Tsetserleg.

Dim. $1,40 \times 0,5 \times 0,25 \mathrm{~m}$.

(Phot. J. Magail.)

Fig. 5

Bouclier et hache gravés sur le côté d'une stèle située à $34 \mathrm{~km}$ à l'est de Gol Mod.

(iranit.

(Phot. J. Magail.)




défunt dans lequel s'enfonce le monolithe. Sur la stèle 1, une frange gravée au ras du sol suggère une séparation entre le domaine inférieur et l'espace terrestre (fig. 3b).

Des motifs géométriques figurent fréquemment entre les thèmes iconographiques. Ils correspondent à une coupure nette, qui semble marquer plus fortement le partage de l'univers. Ainsi, les astres et les cerfs sont quelquefois séparés par un réticulé, ou par une ligne horizontale de cupules (fig. 4 et fig. 8). Parfois même, deux traits parallèles horizontaux, reliés par une série de traits verticaux ou inclinés, donnent au motif l'aspect d'un galon qui entoure la stèle (fig. 6).

Une de ces franges, gravée sur la stèle 4 de Gol Mod, présente un motif de résille (fig. 1b). On peut y voir un cerf de petite taille la traversant de bas en haut, de la partie inférieure au secteur des armes, représenté par un bouclier. Cette évocation du passage de l'animal d'un monde à l'autre constitue peut-être une des clés de la signification de cet art. À la base des monuments, de petits cervidés simulent une ascension en se détachant de l'espace

Fig. 8

Pierre à cerfs

de Daagan Del.

Granit rose.

Dim. 2,5 x $0,39 \times 0,16 \mathrm{~m}$.

(Schéma J. Magail.)

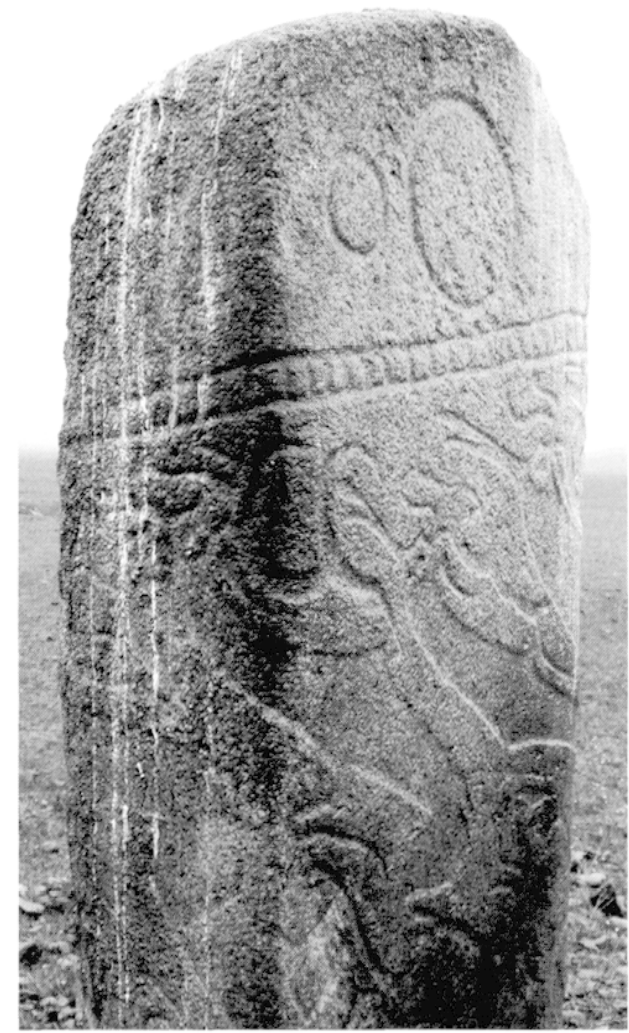

Fig. 6

Stèle 1 de (iol Mod (voir fig. 3).

Détail du sommet.

(Phot. J. Magail.)

sépulcral, le corps élancé vers le ciel. La différence de taille des cervidés entre la base, le centre et le sommet de la stèle 4 , joue sur la perception des distances et donne un effet de circulation aux animaux. Les grands cerfs semblent par exemple plus proches de l'observateur. Cette expression du mouvement garantirait-elle à la stèle son rôle d'axe intermédiaire?

La place et la typologie des cervidés permettent de comprendre leur éventuelle fonction psychopompe. Ils plongent par hordes dans le monde souterrain pour resurgir parfois de l'autre côté en direction du domaine céleste ${ }^{7}$. Dans leur course effrénée, leurs pattes sont repliées sous un corps étiré, en pleine détente. Un museau très allongé, semblable à un bec, prolonge leur silhouette ${ }^{\circledR}$. À ces caractéristiques s'ajoute leur tête arrondie marquée en son centre d'un cil rond, renforçant ainsi l'apparence d'un oiseau (fig. 7).

La surface apparente de chaque bloc de granit a sans doute été étudiée par les auteurs avant de procéder à la composition des ensembles iconographiques. Les gravures sont proches les unes des autres et ne se superposent que très rarement à leurs extrémités. Les formes harmonieuses des cerfs s'imbriquent les unes dans les autres.



Fig. 7

Sommet d'une stèle

située à $\mathbf{4 0} \mathrm{km}$ au sud-ouest de (jol Mod. (Phot. J. Magail.)

L'extrémité des ramures d'un animal se confond parfois avec les andouillers de celui qui le suit dans sa course (fig. 8). Il arrive également que des cuisses ou des pattes s'estompent à la faveur d'une croupe située au-dessous. L'ajustement des figures a vraisemblablement nécessité un tracé préalable. Sur cette roche granitique très dure, une percussion lancée avec percuteur a permis l'exécution définitive de la gravure. Un polissage a parfois été appliqué sur certaines figures, notamment sur le grand cercle de la stèle 5 de Gol Mod (fig. 20). Le style, les thèmes et leur distribution présentent de telles similitudes d'une stèle à l'autre que de véritables écoles ont pu être mises en place. Les graveurs ont strictement observé les mêmes règles en laissant très peu de place à leur créativité individuelle. Ces monuments, répartis sur un si vaste territoire, témoignent donc d'une cohérence cultuelle étonnante.

L'iconographie des cervidés a incité de nombreux chercheurs à entamer une étude comparative entre les diverses cultures nomades des steppes. Fn effet, plusieurs détails se retrouvent dans l'art mobilier du

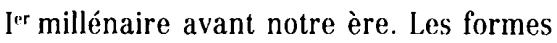
arrondies des ramures déployées le long de la croupe, associées aux pattes repliées 


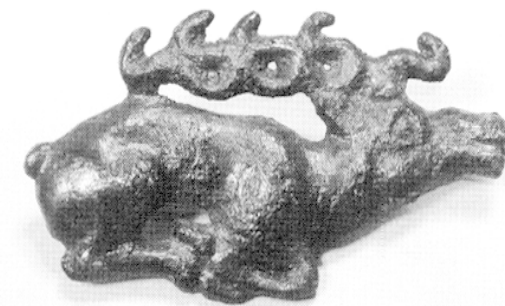

Fig. 9

Applique en forme de cerf.

Bronze, L. $6,8 \mathrm{~cm}$.

Sibérie, région de Minusinsk, ves. av. J.-C.

Musée Guimet, Paris, MA 1170.

(Phot. musée Guimet.)



Fig. 10

Ornement central d'un bouclier

Or, 1. $31.7 \mathrm{~mm}$,

kourgane de Kostromskaïa.

Musée de l'Ermitage, Saint-Pétersbourg

(Schéma B. Sassatelli.) sous le ventre, sont les principaux points de ressemblance. Les nombreuses appliques en bronze de la culture de Tagar du sud de la Sibérie (Minusinsk) présentent ainsi des similarités stylistiques avec les «pierres à cerfs» de Mongolie (fig. 9). Il est probable que leur dépôt dans les sépultures les liait symboliquement avec le devenir des défunts. I.a comparaison peut s'étendre aux

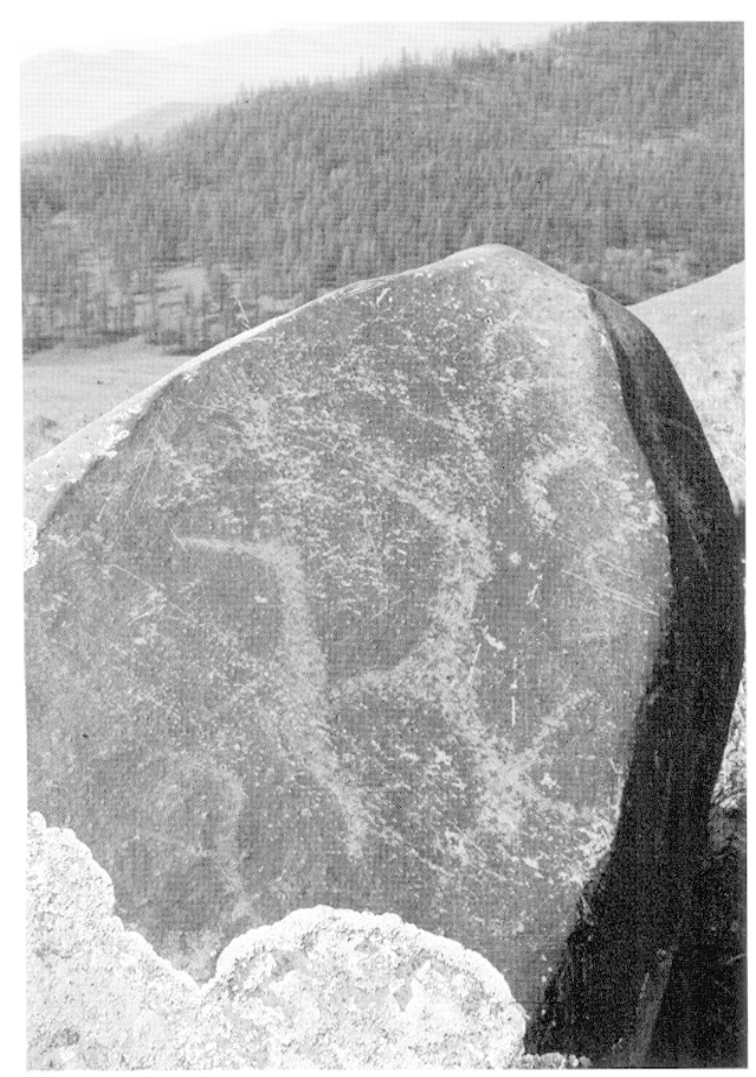

Fig. 12

Pierre gravée de trois cervidés et d'un cavalier.

Dim. $50 \times 40 \mathrm{~cm}$, à $6 \mathrm{~km}$ de Gol Mod.

(Phot. J. Magail.) cultures scythes du Caucase, éloignées de milliers de kilomètres à l'ouest (fig. 10): moulés sur les ceintures et fixés sur les boucliers, ces cervidés ornaient les attributs des guerriers tout en les protégeant peut-être de la défaite et de la mort.

Limage du cervidé n'avait sans doute pas exactement la même signification d'un bout à l'autre de la steppe, mais elle était liće à la dévotion envers les esprits de la nature. Aussi, les gravures de cerfs sur ces stèles sont-elles à rapprocher de certaines pratiques rituelles, car elles sont associées à des lieux cultuels constitués d'enclos rectangulaires, de tumulus et de cercles de pierres sèches. A Gol Mod, la stèle 4 est située à $100 \mathrm{~m}$ d'un grand enclos cultuel marqué en son centre par un tumulus de pierre. On notera également que les autres stèles répertoriées le long des vallées de la Hunuin Gol et du Tamir, vers le sud, sont également proches de vestiges de centres cultuels.

Dans cette optique, on peut supposer que certains animaux ont eu le rôle de médiateur entre les hommes et le monde surnaturel de l'au-delà. Ainsi, dans l'Altaï kazakhstanais, un prince Saka a été inhumé au III" $s$. avant notre ère avec 13 chevaux munis de cornes factices: les chevaux sacrifiés découverts dans ce kourgane princier du site de Berel'par la Mission archéologique française en Asie centrale, étaient équipés de paires de cornes en bois, imitant notamment celles du bouquetin". Des vestiges comparables, conservés au musée de l'Ermitage de Saint-Pétersbourg, ont été retrouvés dans les sépultures de Pazyryk. Lors des funérailles chez les Scythes de l'Altaï et de Sibérie du Sud, le cheval semble être devenu le passeur domestiqué qu'il fallait grimer pour réintégrer le bestiaire originel. Quant aux «pierres à cerfs»,



Fig. 11

Scène de poursuite d'un cerf par un canidé. Gravure rupestre, âge du bronze. site de Baga Oigor III, Mongolie de l'Ouest. (I)'après Jacobson, Kubarev et Tseevendorj 2001. vol. Texte et Figures, $n^{\circ}$ 913.)

E. Novgorodova a pu observer que, dans leur dernière phase stylistique, le cheval remplace petit à petit les cervidés ${ }^{16}$.

Les religions steppiques considéraient les mouflons, les ibex, les bouquetins et les cerfs comme des entités animées par les esprits de la nature, qui guidaient leurs déplacements annuels. La perte et la pousse de leurs bois, en phase avec les saisons et les mouvements cosmiques, renforçaient sans doute l'aspect mythique de ce gibier traqué par les groupes de nomades ${ }^{11}$. Les nombreuses scènes de chasse gravées sur les pans rocheux de la région ouest de la Mongolie témoignent de la continuité de ces pratiques, de l'âge du bronze à la période turque ${ }^{12}$. La typologie de certains cervidés pourchassés qui y sont représentés (fig. 11) correspond tout à fait à celle des "pierres à cerfs», montrant ainsi que les monuments funéraires et cultuels n'avaient pas l'exclusivité de cet art ${ }^{13}$. En effet, les «cerfs à bec» apparaissent à l'âge du bronze final au centre de certaines scènes rupestres, parfois entourés d'archers et de chiens.

L'art rupestre en Mongolie fait apparaître bien d'autres types iconographiques de cervidés. Pour exemple, sur une roche située à $6 \mathrm{~km}$ à l'ouest de Gol Mod, trois animaux suivis d'un cavalier semblent composer une scène de chasse (fig. 12). De style très différent de celui des "pierres à cerfs", le cerf, la biche, le faon et leur poursuivant sont très effacés. Ces figurations d'animaux, qui nécessitent une expertise, possèdent les caractéristiques de la période du début de l'âge du bronze.

Dès 1962, N. L. Tchlenova qui rappelait les ressemblances entre les appliques métalliques de cervidés et les gravures rupestres, constatait également le lien entre 


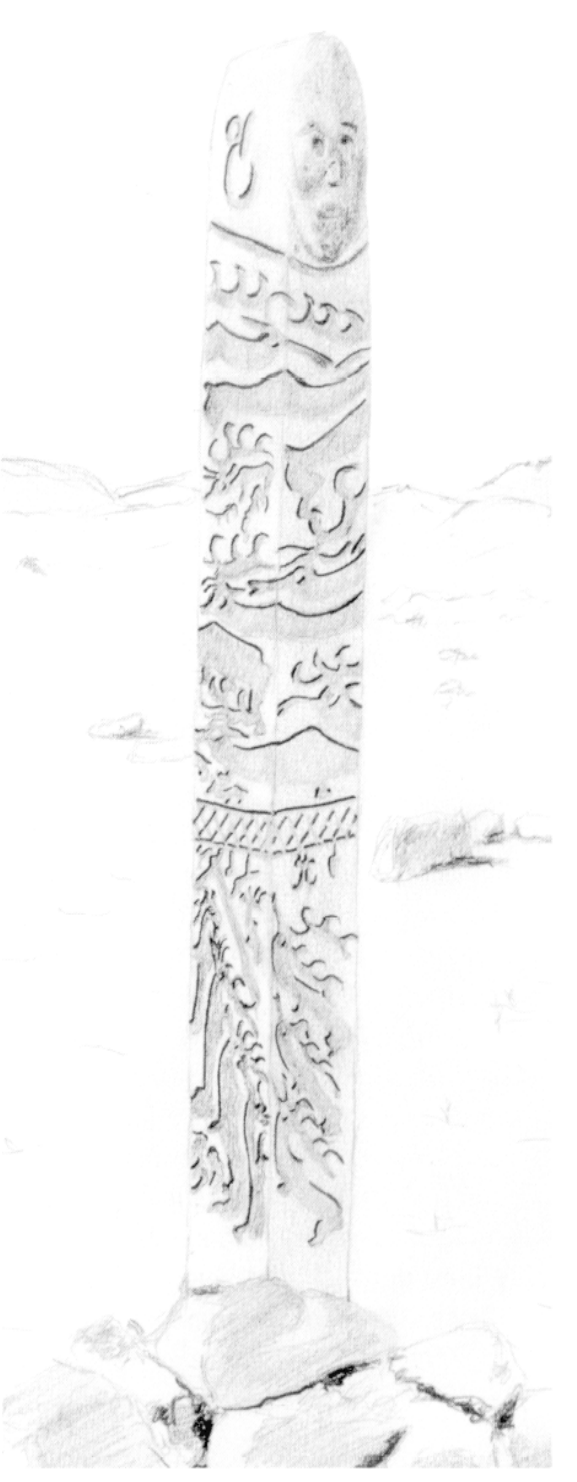

Fig. 13

Stèle de Uushig.

Granit blanc, 2,3 x 0,6 x 0,4 m,

Uushig, province de Hövsgöl, soum de Mönör. (Schéma B. Sassatelli.)

les "pierres à cerfs" et le monde des guerriers $^{14}$. L'auteur attirait entre autre l'attention sur certaines "pierres à cerfs" dont le sommet comporte, sur une face, l'ébauche d'un visage. Ces pierres, peu nombreuses, se situent notamment dans la province mongole de Hövsgöl (fig. 13) et dans la région russe de Touva. Selon N. L. Tchlenova, semblables à des statues sans bras ni jambes, ces pierres ont une iconographie qui peut être assimilée aux trois parties du corps d'un guerrier: la tête, le buste et la ceinture. Ainsi, les cercles gravés au sommet seraient les boucles d'oreilles du personnage, la frange au niveau de son cou serait son collier, et la frange à la base du monument représenterait la ceinture où sont suspendues ses armes. Les traits gravés entre les armes, à la ceinture, suggèrent effectivement des crochets dont I). Savinov a retrouvé les pièces archéologiques correspondantes ${ }^{15}$. Enfin, pour E. Novgorodova, les visages ont bien été sculptés en même temps que les gravures, dont le style renverrait aux stèles les plus anciennes ${ }^{16}$.

C.es «pierres à cerfs» anthropomorphes représentent incontestablement une catégorie spécifique qui contribue à la compréhension de ces monuments. Fichés en terre, les monolithes pourraient évoquer un guerrier cosmique dont les différentes parties du corps se rapporteraient aux secteurs de l'univers. Cette hypothèse fait référence aux mythologies qui personnifient l'axe cosmique sous les traits d'un être divin, parfois même d'un géant. Chez. les nomades du $I^{\text {rr }}$ millénaire, il aurait pu prendre l'allure d'un guerrier, héros fondateur des diverses tribus. Chez les sédentaires han, c'étaient des représentations d'arbres cosmiques en bronze qui étaient placées sur les sépultures, afin de permettre à l'âme du défunt de rejoindre le ciel.

Sur la trentaine de monuments répertoriés en 2004 dans les vallées de la Ilunuin Gol et du Tamir, aucun ne présente de visage en son sommet. Gravées de poignards, de haches, d'arcs et de boucliers, les stèles de Gol Mod sont placées sur des tombes qui n'ont pas encore été fouillées. Toutefois, les personnages inhumés dans ce type de sépulture sont en principe ensevelis avec leurs propres armes (fig. 14) ${ }^{17}$. Une partie des travaux de $\mathrm{k}$. Novgorodova a porté sur la comparaison entre les pièces archéologiques et leur figuration sur les stèles. Selon sa classification, les armes gravées à Gol Mod (fig. 15) sont du type de Karasuksk.

Le bout de la lame des poignards des stèles 1 et 2, en forme de trapèze (fig.16),
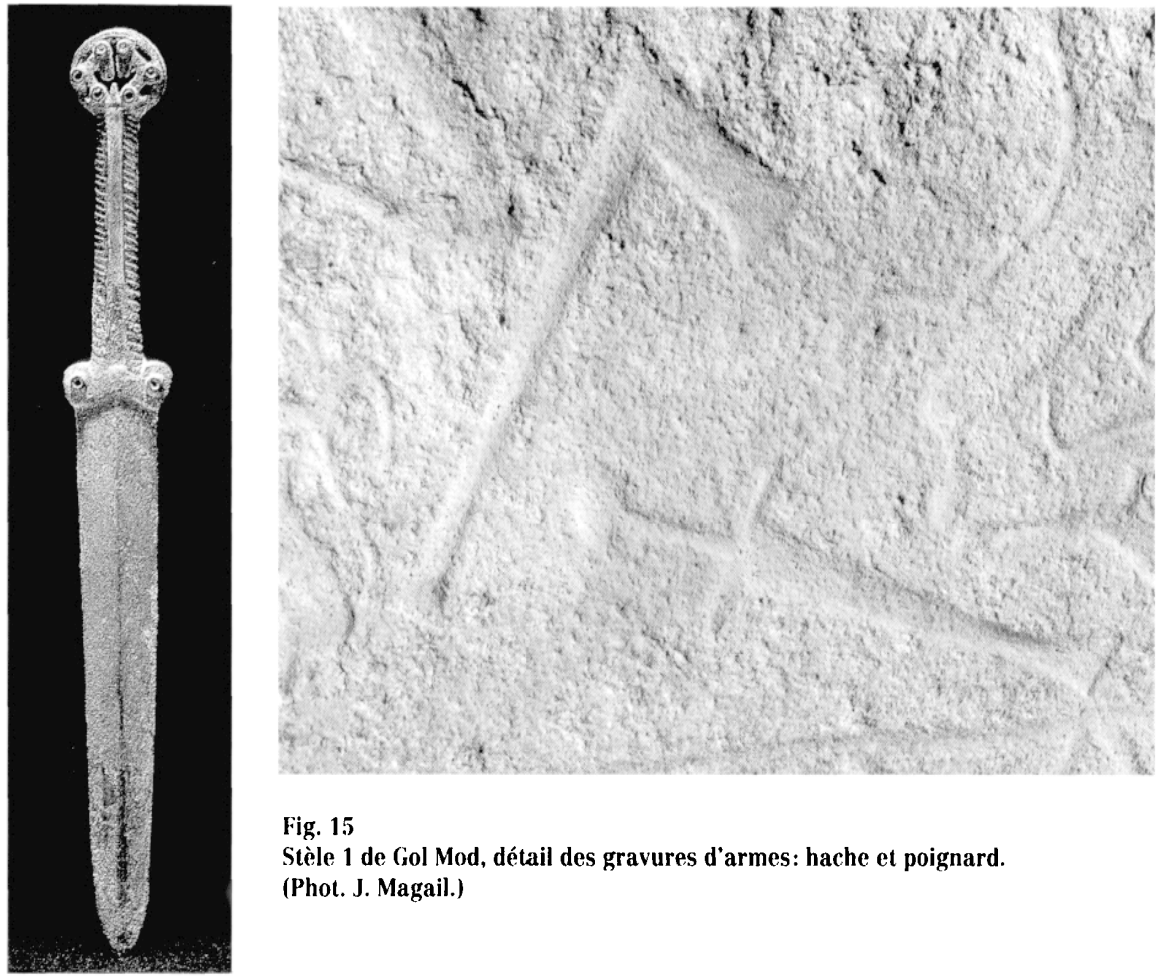

Fig. 15

Stèle 1 de Gol Mod, détail des gravures d'armes: hache et poignard. (Phot. J. Magail.)

Fig. 14

Épée courte.

Bronze. L. $26 \mathrm{~cm}$.

Chine du Nord,

ves. av. J.-C.

Musée Guimet, Paris, MG 20782.

(Phot. musée Guimet.)

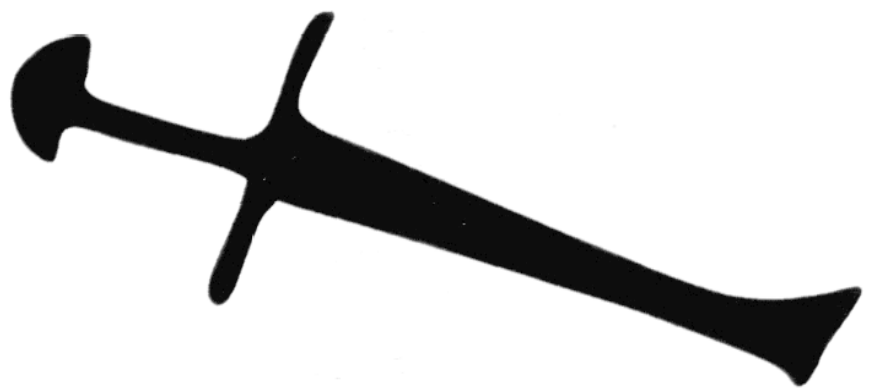

Fig. 16

Stèle 1 de Gol Mod, schéma de la gravure du poignard. (Schéma J. Magail.) 


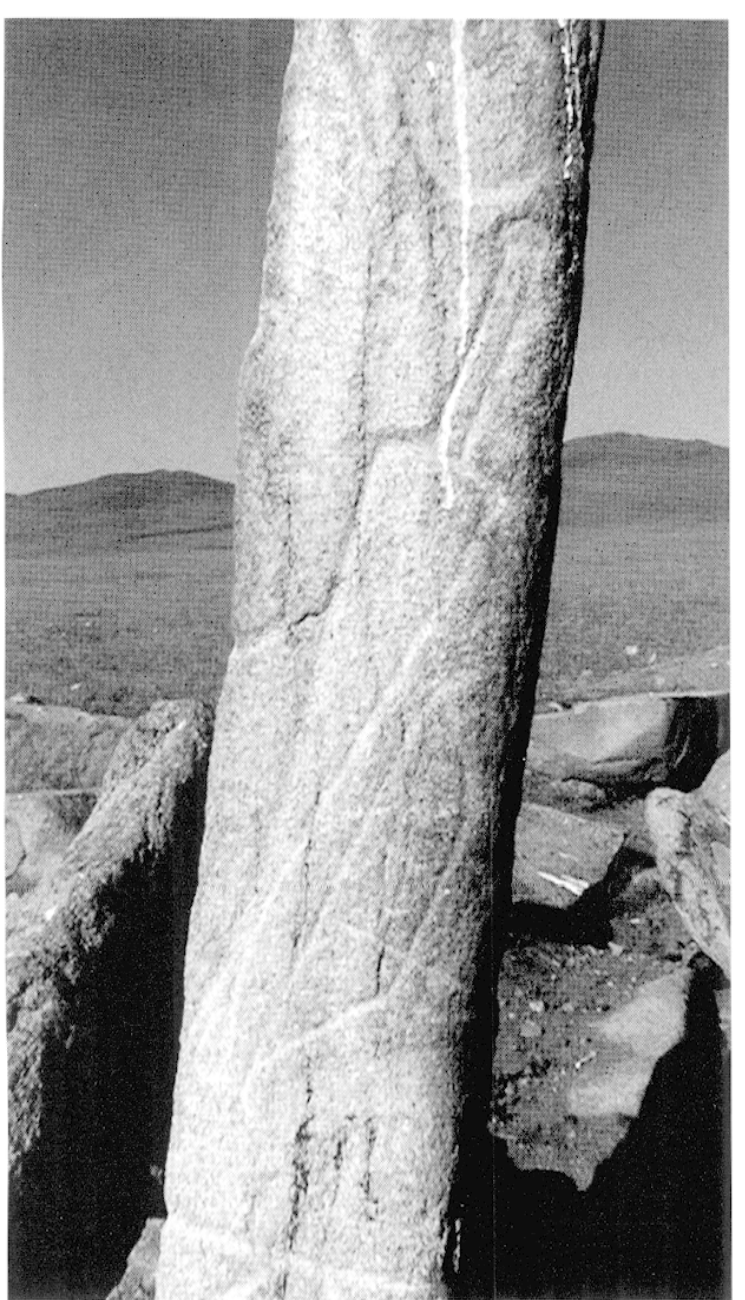

Fig. 17

Stèle 2 de Gol Mod,

détail des gravures d'armes: épée courte et arc. (Phot. J. Magail.)

indique qu'ils sont représentés dans leur fourreau ${ }^{18}$. D'un style plus schématique que les figurations de cerfs, les poignards, la hache et l'arc sont figurés au-dessus des franges géométriques, et ne sont donc pas suspendus à la ceinture (fig. 15 et 17). Grâce à certains détails iconographiques, il est d'ailleurs possible d'identifier le type de hache gravée: à Gol Mod et dans ses environs, ce sont des haches emmanchées grâce à des systèmes à douille. L'emmanchement est soit perpendiculaire, soit dans l'axe de la lame (fig. 15). Deux stèles, l'une à $34 \mathrm{~km}$ à l'est et l'autre à $74 \mathrm{~km}$ au sudouest de Gol Mod (site de Tsatsyn Ereg), comportent chacune une représentation de hache avec un anneau au dos de la lame (fig. 18).

Au-delà de leur fonction première, les armes transcendent le champ social et religieux. En premier lieu, elles sont des objets d'apparat et de prestige, arborées par les chefs de clan. En second lieu, leur éventuel rôle magique pouvait canaliser les puissances surnaturelles susceptibles d'intercéder en faveur de leurs possesseurs. Les superbes hallebardes de la culture de Tagar possèdent par exemple, à la base de leur lame, une statuette d'animal moulée dans la masse. L'utilisation rituelle ou cérémonielle de telles armes est une hypothèse séduisante, étayée de fait par leur fragilité difficilement conciliable avec les puissantes luttes guerrières. On peut en dire autant des poignards gravés sur certaines «pierres à cerfs" dont le pommeau est en forme de tête de cervidé. L'animal sauvage de la grande steppe accompagnait les guerriers, de leurs premières luttes à leur sépulcre. Ce bestiaire porté sur soi, quelquefois même tatoué (Pazyryk et Ukok), avait probablement la fonction symbolique, tout aussi indispensable que le tranchant des lames, de rendre invincibles les hommes au combat ${ }^{19}$.

Les affrontements de mouflons et les félins agrippant leur proie sont des thèmes également très répandus chez les populations d'Asie centrale et de Haute Asie, comme en témoignent leurs nombreuses représentations sur les boucles de ceinture. Les mêmes scènes de prédation se retrouvent sur le tapis découvert dans une tombe Xiongnu de Noïn Oula" ${ }^{1 "}$. Sur les "pierres à cerfs», des félins sont parfois représentés, même si les stèles de Gol Mod n'en comportent pas. Beaucoup plus discrets que les grands cervidés et souvent placés autour du soleil, ils ont suscité chez E. Novgorodova l'hypothèse d'une représentation de l'ancien mythe du rapt du soleil par les animaux.

I.a période des «pierres à cerfs» constitue une phase de transition entre des sociétés tribales et une civilisation Xiongnu très organisée, capable de réunir un grand numbre de combattants face aux Han. Si la valorisation du guerrier a subsisté, la pratique de l'élevage extensif a vraisemblable-



Fig. 18

Hache gravée sur une stèle couchée. site de Tsatsyn Ereg,

$74 \mathrm{~km}$ au sud-ouest de Gol Mod.

(Phot. J. Magail.)

ment engendré des modifications démographiques et un changement du rapport entre l'homme et son milieu. La société nomade s'est alors hiérarchisée de façon plus significative et a adopté de nouvelles stratégies politiques et religieuses. Chez les Xiongnu, rapporte Sima Qian, l'empereur, élu parmi les meilleurs guerriers, devenait le «fils du ciel et de la terre» et l'incarnation du soleil et de la lune devant lesquels il se prosternait matin et soir ${ }^{21}$. Il était le combattant suprême de tous les clans, celui dont la sépulture devenait un lieu de culte. En effet, l'architecture des tombes aristocratiques Xiongnu comporte une terrasse et une allée funéraire aménagées à des fins rituelles; ces deux éléments sont systématiquement orientés vers le nord, suggérant un lien avec des objets célestes ${ }^{22}$. La découverte, par la Mission archéologique française en Mongolie, d'un soleil et d'une lune en fer dans une tombe Xiongnu du site d'Egiin Gol (fig. 19) abonde dans le sens des écrits de Sima Qian. Ces pièces fixées sur les parois du cercueil, trouvent un écho au sommet des "pierres à cerfs» où sont gravés les deux astres (fig. 6 et 20). À la mort 
du nomade, son corps devenait en quelque sorte sédentaire tandis que son âme poursuivait son voyage. Finfin, l'agencement dans l'espace des supports de cet art funéraire et cultuel permettait vraisemblablement de faciliter le cheminement de l'âme ${ }^{23}$. Pour l'heure, rien ne permet de supposer que l'orientation actuelle des stèles de (iol Mod était différente à l'âge du bronze.

La cosmologie suggérée par l'étagement des armes, des cerfs au museau allongé et des astres, nécessite la recherche du modèle religieux à l'origine de cette expression artistique ${ }^{24}$. En Haute Asie, l'iconographie des cervidés est immergée dans un système de croyances, dont l'un des thèmes centraux est la circulation des âmes et des esprits. Certes, 2500 ans après l'édification des «pierres à cerfs". les rituels observés par les ethnologues ne peuvent être transposés tels quels aux populations de l'âge du bronze. Toutefois, les vestiges archéologiques funciraires du $\mathrm{I}^{\mathrm{r}}$ millénaire avant notre ère évoquent un contexte religieux profondément marqué par l'animisme. Rien n'indique qu'il existait un chamane dans les sociétés Saka ou dans les tribus qui érigeaient les «pierres à cerfs", mais le chamanisme, qui n'est qu'une déclinaison de l'animisme, comporte des éléments susceptibles d'être com- parés à ceux des monuments ornés. Le chamane lui-même, médiateur entre les hommes et les esprits, porte une «cuirasse» ornée d'oiseaux, de figurines et d'astres susceptibles de l'aider dans son voyage cosmologique. Le rôle apotropaïque de ces figures métalliques accrochées à ses vêtements donne une idée de la fonction que peuvent revêtir de telles iconographies.

Au sein des différentes ethnies sibériennes, le taureau, le cervidé, l'élan et le renne sont des animaux auxiliaires que le chamane imite lors de ses rituels par des sauts et des trépignements. $\dot{A}$ ces occasions, il porte parfois une coiffe ornée de cornes factices en métal, grimée comme les chevaux de Pazyryk. La couronne métallique Evenke dotée de cornes en fer, rapportée par Joseph Martin en 1887 (fig. 21), ne laisse aucun doute sur l'imitation du cervidé2. Le costume du chamane est une véritable armure et ses ramures métalliques servent à se battre contre les esprits ennemis tel «le cervidé étalon défendant

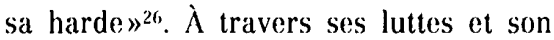
mariage avec la fille des esprits, le chamane renvoie une image virile qui appartient au registre du guerrier. Son rôle de médiateur entre les chasseurs et les esprits de la nature révèle les mécanismes symboliques de ce modèle religieux ancestral.



Fig. 21

Coiffe de chamane (calotte et couronne) Evenk, Sibérie orientale. Musée de l'Homme. M.II. 87.42 .2 (1) \& M.H. 87.42.2(2) (I)'après Beffa et Delaby 1990 (1) Publications Scientifiques du Muséum national d'histoire naturelle, Paris.)

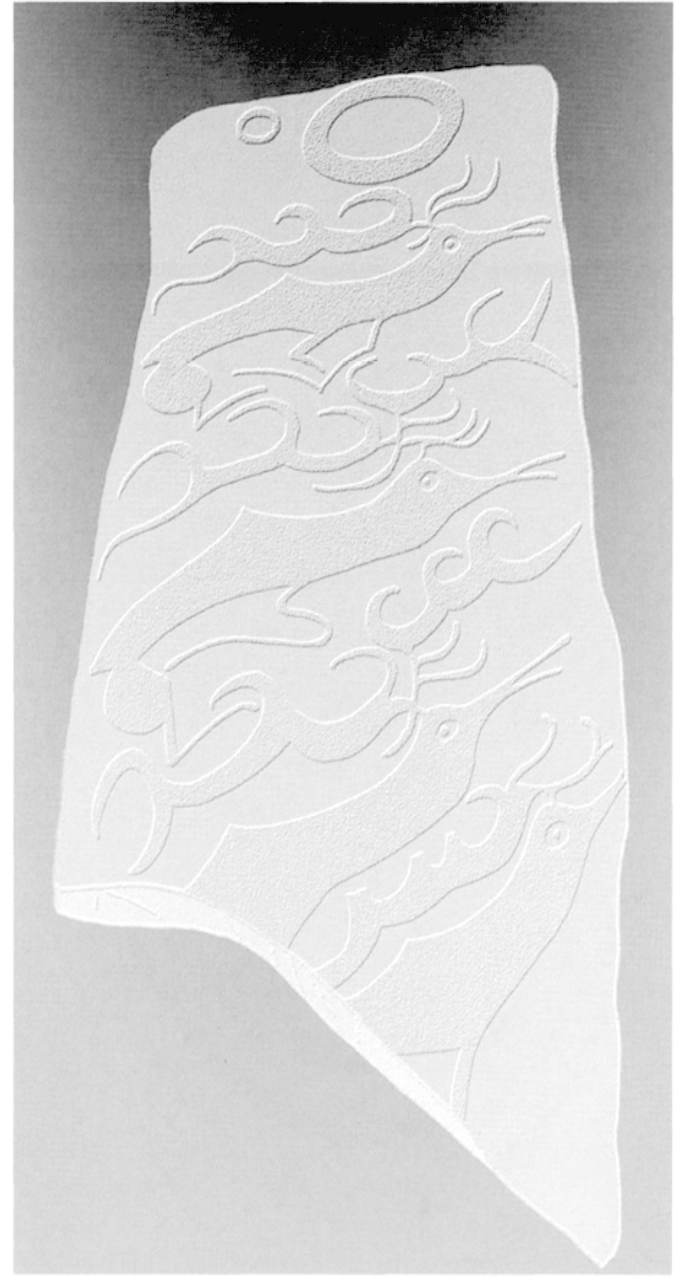

Fig. 20

Stèle 5 de Gol Mod, cassée et couchée sur le sol. (iranit blanc.

(Schéma J. Magail.)

Les cervidés en forme d'oiseaux des "pierres à cerfs" pouvaient aussi avoir le rôle d'intermédiaire entre la steppe et le ciel. I.eur envol peut ainsi être comparé à l'envol du chamane en partance vers l'autre monde. C'est bien du déplacement dont il est surtout question, car il est fondamental dans le nomadisme. À ce sujet, la position et l'expression des cerfs des "pierres à cerfs» s'inscrivent parfaitement dans l'art des steppes, qui prend toute sa signification dans le mouvement. La lecture des stèles 1 et 4 de ciol Mod met en évidence un va-et-vient des cervidés entre le haut et le bas. Sur le site de Tsatsyn Freg, le même principe s'applique sur une stèle ornée de six animaux qui est aujourd'hui renversée et très abîmée (fig. 22a-b): trois cerfs, gravés de part et d'autre du monument, ont un mouvement opposé. Une triade montait donc vers le ciel, tandis que l'autre descendait vers le sol.

la suggestion du mouvement n'induit pas pour autant un art narratif, elle est davantage l'élément central d'une cosmo- 
logie liće au cycle de la nature et à la circulation des esprits. Les sépultures, les tumulus et les cercles de pierres parmi lesquels sont implantées les «pierres à cerf's" montrent la complexité de l'agencement de chaque lieu. C'est donc la poursuite des recherches archéologiques sur des sites comprenant ces monuments qui permettra de mieux comprendre le contexte cultuel de ce patrimoine exceptionnel.

JÉROMME MACAII.

Co-directeur de l'Expédition archéologique en Mongolie du Musée d'Anthropologie préhistorique de Monaco

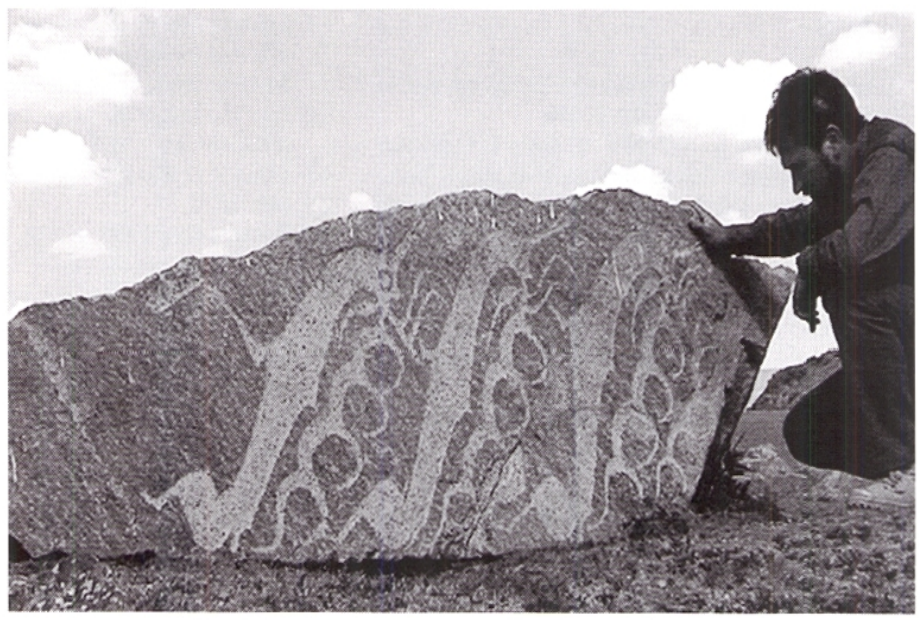

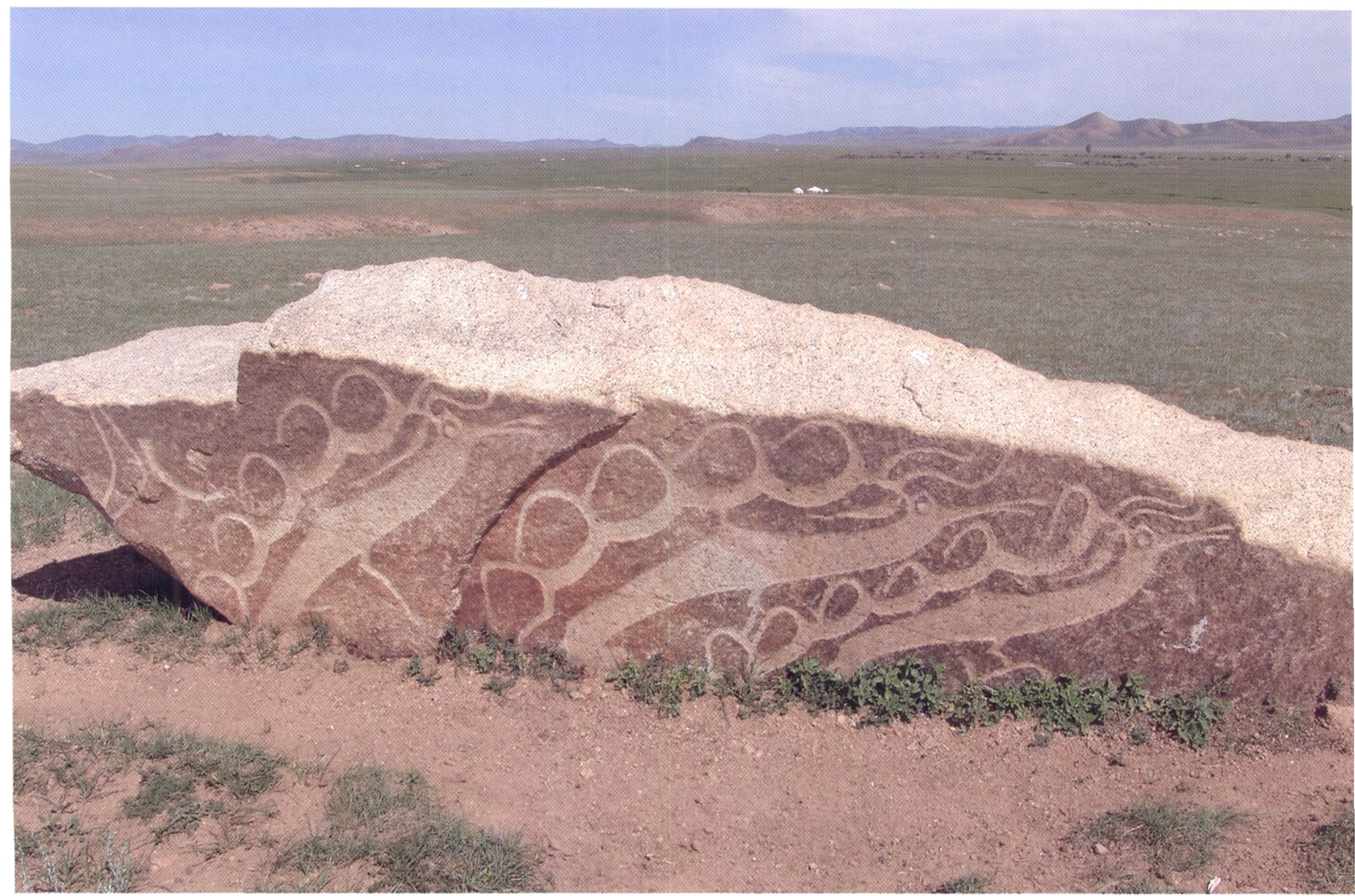

Fig. $22 a-b$

Stèle brisée, site de Tsatsyn Ereg.

Côtés sud et nord de la stèle, gravés chacun de trois cerfs progressant en sens inverse.

(Phot. J. Magail.) 
Notes

1 Le professeur D. Tseevendorj a étudié ces monuments: Tseevendorj 1999, p. 295.

2 Magail 2003, p. 182-208. Je tiens à remercier Yerööl Frdene, Juliana Holotova et Indira Yakovenko pour l'aide efficace qu'ils m'ont apportée par leurs traductions des textes russes et mongols.

3 Les cartes d'E. Novgorodova montrent que les «pierres à cerfs" sont réparties au centre du territoire de l'empire Xiongnu: Novgorodova 1989. p. 181.

4650 sont réparties sur le territoire mongol et une cinquantaine dans les régions limitrophes. Tseevendorj 2002, p. 101

5 Hypothèse formulée par le professeur D. Tseevendorj, voir Tseevendorj 1999. E. Novgorodova évoque également le caractère solaire des cercles rayonnants et de la forme des ramures des cervidés, Novgorodova 1989, p. 208.

6 Tseevendorj 2003.
7 Sur un relevé de D. Savinov, 14 cervidés en pleine ascension occupent une face du monument, voir Savinov 1994, illustration de couverture.

8 Pour k. Novgorodova, ce sont des cerfs à bec qui prennent leur envol, Novgorodova 1989, p. 187.

9 Samachev, Bazarbaeva, Zhumabekova et Francfort. 2000 .

10 Novgorodova 1989, p. 212.

11 Sima Qian précise qu'à l'époque Xiongnu, les guerriers s'entraînaient lors de chasses collectives. Shiji, 110, p. 2888

12 Jacobson, Kubarev et Tseevendorj, 2001

13 Ibid. Vol.1: p. 275, 382, 464. - Vol. 2: p. 48, 59, 164.

14 Tchlenova 1962.

15 Savinov 1994, p. 198

16 Novgorodova 1989, p. 210

17 Kubarev 1996

18 Les fourreaux des poignards pouvaient être en bois comme ceux découverts dans l'Altaï. Molodin 1996.

19 Les premiers tatouages d'animaux à cornes ont été retrouvés sur un défunt de Pazyryk en 1947. le corps tatoué d'une jeune femme, exhumé de sa tombe située sur le plateau d'Ukok dans l'Altaï. comporte la même iconographie, voir Polosmak 1996.

20 Rudenko 1962, p. 57, et Tables XXXIX-XLV

21 Shiji, 110, 2892-2899.

22 Magail 2003.

23 Novgorodova 1989 , p. 204. Sur certains sites mongols, tels que ceux de Uushig, Shatar Chuluu et Bodonchyn Gol, plusieurs "pierres à cerfs" sont disposées en deux rangées afin de constituer une allée.

24 Plusieurs auteurs ont évoqué le lien entre l'art rupestre des steppes et une religion chamanique. Voir: "Tombes gelées de Sibérie». $\mathrm{N}^{\circ}$ spécial de Dossiers d'archéologie, Éditions Faton, 1996. $\mathrm{N}^{\circ} 212$, et notamment Devlet 1996 où des gravures de masques cornus de l'âge du bronze sont rapprochées de rituels chamaniques.

25 Beffa et Delaby 1999.

26 Hamayon 1990, p. 662

\section{Bibliographie}

- Samashiv Z. S.; Bazarbativa G.A.; Zhumabikova G.S.; Franchort II.-P., 2000: " le kourgane de Berel'dans l'Altaï kazakhstanais», Arts Asiatiques, vol. 55. p. $5-20$.

- BlFFa M.-L.: Delaby L.. 1999: «Festins d'âmes et robes d'esprits. Les objets chamaniques sibériens du Musée de l'Homme", Mémoires du Muséum national d'Histoire naturelle, tome 181, p. 87-93.

- Di:lacour C., 2001 : De bronze, d'or el d'argent. Arts somptuaires de la Chine, Paris, Réunion des musées nationaux.

- Devilet A. Marianne, 1996: «Murgur-Sargol, sanctuaire de l’âge du Bronze sur l'lénisséim Dossiers d'archéologie, $\mathrm{n}^{\circ} 212$, p. $58-63\left(\mathrm{~N}^{\circ}\right.$ thématique: "Tombes gelées de Sibérie»)

- FRANCFORT H.-P., 1996: «Art rupestre et tombes gelées», Dossiers d'archéologie, $\mathrm{n}^{\circ} 212$, p. $2.9\left(\mathrm{~N}^{\circ}\right.$ thématique: «Tombes gelées de Sibérie»)

- JacoBson E., 1993: The Deer Goddess of Ancient Siberia: A Study in the Ecology of Belief, Leiden. Brill Academic Publishers.

- Jacobson E.; Kubariv V.; TshivindorJ D., 2001. Répertoire des pétroglyphes d'Asie Centrale. Fascicule $n^{\circ}$ 6: Mongolie du Nord-Ouest, Tsagaan Salaa Baga Oigor, Paris, De Boccard, 2 vol.: Planches, et Texte et Figures (Mémoires de la Mission archéologique française en Asie Centrale, tome V/6)

- Hamayon R., 1998: "L'épingle du jeu». Revue du M.A.U.S.S. [Paris, Ia Découverte/M.A.U.S.S.] $\mathrm{n}^{\circ} 12$, $2^{2}$ sem., p. 103-121 ( $\mathrm{N}^{\circ}$ thématique: Plus réel que le réel, le symbolisme).

- Hamaron R., 1990: La chasse à l'âme. Esquisse d'une théorie du chamanisme sibérien. Nanterre |Paris], Société d'ethnologie.

- KubarFV V.D., 1996: "Lart antique des montagnes d'or", Dossiers d'archéologie, $\mathrm{n}^{\circ} 212$, p. $42-57\left(\mathrm{~N}^{\circ}\right.$ thématique: "Tombes gelées de Sibérie»)

- Kubarl:V V.ID., 1979: Drevnie Izvayuniya Altaya,

Novosibirsk, Olennie kamni.

- Macial. J., 2003: Chapitre «Entre steppe et ciel», in Mongolie, le premier empire des steppes, Arles. Actes Sud \& Mission archéologique française en Mongolie, p. 182-208.

- Maciall. J., 2004: "Mongolian Stag stones", International Newsletter On Rock Art, $\mathrm{n}^{\circ}$ 39. p. 17-27.

- Macill. J., 2005: «Les "pierres à cerfs" des vallées Hunuy et Tamir en Mongolie», Bulletin du Musée d'Anthropologie préhistorique de Monaco, $\mathrm{n}^{\circ} 45$. Monaco. p. 41-56.

- Miniais S., 2002: "Les Huns en Sibérie: découvertes et problèmes", Dossiers d'archéologie, $n^{\circ} 270$, p. 62 69 ( $\mathrm{N}^{\circ}$ thématique: «Tombes gelées de Sibérie»).

MoloDIN V.I., 1996: "Un kourgane gelé d'époque scythe», Dossiers d'archéologie, $\mathrm{n}^{\circ}$ 212, p. 36-41 ( $N^{\circ}$ thématique: "Tombes gelées de Sibérie»)

- Novgoronova Ł. A., 1989: Drevnyaya Mongoliya, Moskva, Nauka.

- Polosmak Natalia B., 1996: «La prêtresse altaïque». Dossiers d'archéologie, $\mathrm{n}^{\circ} 212$, p. 28-35 ( ${ }^{\circ}$ thématique: «Tombes gelées de Sibérie»)

- Rudrino S. I., 1970: Frozen Tombs of Siberia. The Pazyryk Burials of Iron-Age Horsemen, London, J.M. I)ent \& Sons I.td

- Rtır.nko S. I., 1962: Kultura Khunnov i noinulinskie kurgany, Moskva/Leningrad, Isdatelstvo Akademii Nauk SSSR.

- SAVINov D. G., 1994: Olennie kamni v kulture kotchevnikov Evrasii (Les pierres à cerf dans la culture des nomades d'Eurasie]), S.-Peterburg, Izdatelstvo S.-Peterburgskogo universiteta.
- ScHIITZ V., 1985: «Les Scythes et le monde des steppes", Le grand atlas de l'archéologie, Paris, Encyclopadia Universalis, p. 210-225.

- Sнек Y.A. 1980: Ranniy elap skifu-sibirskogo zverinogo stilya, Kemerovo, Skifo-sibirskoe kulturnoistoritcheskoe edinstvo.

- Sima Qian. 1998: Shiji (Mémoires historiques Han). Beijing, ed. Zhonghua shuju.

- TCHLENOVA N.L. 1962: «Ob olennykh kamnyakh Mongolii i Sibiri", Mongolskij arkheologitcheskij sbornik [Akademiya Nauk SSSR], p. 28-35.

- TIVANenko, A.V. 1995: «()lennie kamni Zabaïkaliya», in Kulturi i pamyatniki bronzovogo i rannego jelez. nogo vekov Zabaikaliya i Mongolii, Ulan-Udè, BNT's SO RAN, Rossiyskaya Akademiya Nauk, c. 102.

- TSFEVENibors D). 1979: "Mongol nutgaas oldson zarim bugan khöshöö», Arkheologiin suudlal [Ulaanbaatar], tome VII, fasc. 13.

- Tsfenvendor. D., 1999: Mongolyn ertnii urlagiin tüükh, Ulaanbaatar, Académie des sciences de Mongolie - Institut d'histoire.

- [Tservenook. D., 2002]: Tsellyentor. I).; Bayar D. TSLRENDNGiVA Ya.; OTCHIRKHUYAG Ts., 2002: Mongolyn arkheologi, Ulan-Bator, Shinjlekh ukhaany akademi, Tüükhiin khüreelen, Arkheologiin sudalgaany töv

- TStrivendor.J D., 2003: «Töv Aziin ertnii nüüdeltchdiin bambai", in Archaeological study in Mongolia. Collection of Research Articles and Reports 1973. 1982, Ulaanbaatar, Tome 1, p. 48-52.

- Vol.Kov, V.V., 1981: Olennie kamni Mongolii, Ulaanbaatar, Nautchniy Mir. 